\title{
Secure Data Transaction and Data Analysis of IOT Devices
}

\section{Using Blockchain}

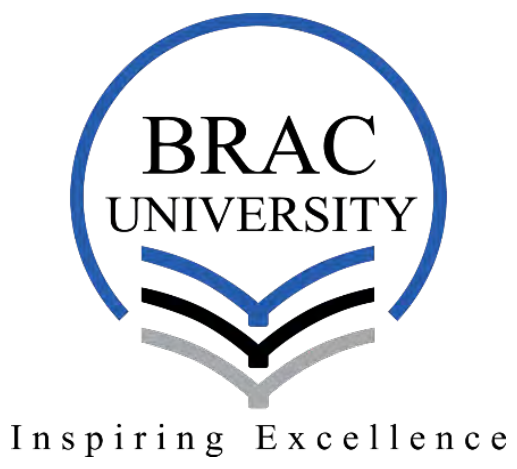

SUBMISSION DATE: 24.12.17

Md. Adib Muhtasim-14101140

Syeda Ramisa Fariha- 14101137

Nabila Islam - 16341024

Rayhan Rashid - 14101106

Department of Computer Science and Engineering

Supervisor:

Dr. Mahbub Alam Majumdar

Professor

Department of Computer Science and Engineering 


\section{Declaration}

We, hereby declare that this thesis is based on results we have found ourselves. Materials of work from researchers conducted by others are mentioned in references.

Dr. MahbubAlamMajumdar

Professor

Department of Computer Science and

Engineering

BRAC University
Md. Adib Muhtasim-14101140

SyedaRamisaFariha- 14101137

Nabila Islam - 16341024

Rayhan Rashid - 14101106 


\begin{abstract}
\end{abstract}
The Internet of Things (IOT) is a network formed by electronic objects which have embedded sensors, software and network connectivity in them. They can collect and exchange and are prone to both cyber-attack and physical tampering. Thus, it is essential to encrypt their communications, by addressing new challenges such as impersonating "things" or nodes, denial-of-sleep attacks that drain batteries, to denial-of-service attacks (DoS). A peer-to-peer model can eliminate the dependency of a centralized data center. A Blockchain is a model for a distributed database of records of all transactions or digital events that have been executed and shared among participating nodes. In it, digital signatures are validated instead of physical signatures. With a decentralized approach implemented through cryptographic algorithms, blockchainoversees a secure transaction betweenIOT devices and coordinate them. This paper explores one of the process of incorporating blockchain with a very vulnerable centralized IOT data transactions. IOT devices are represented as Aircrafts in which the engine data and various data from the aircrafts' sensors are sent to the airports for analysis. After analysis, the analyzed data is sent to nearby airports and also to that particular aircraft. The paper analyzes how the blockchain network is created, the time latency of sending the data of the IOT devices through this blockchain network and some parts of the analysis process which is required for the Aircraft maintenance. 


\section{Acknowledgement}

We would like to express our utmost gratitude and appreciation to our supervisor Dr. MahbubAlamMajumdarfor his attention and time. We would also like to thank him for giving us the opportunity to work on this topic and assisting us throughout the process. Secondly, we would also like to thank our co-advisor MonzurMorshed for his guidance and time. 


\section{TABLE OF CONTENTS}

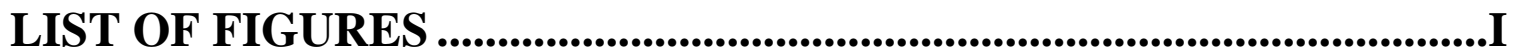

LIST OF ALGORITHMS ...................................................................

CHAPTER 1 LITERATURE REVIEW ............................................. 1

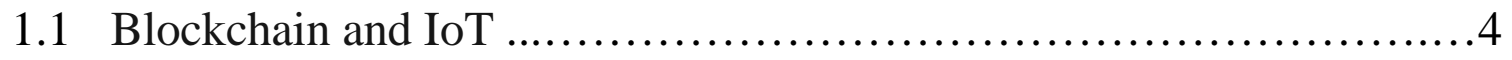

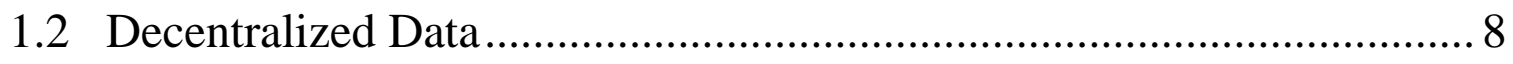

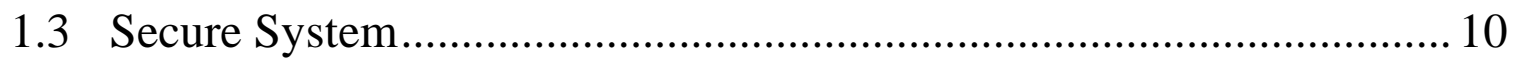

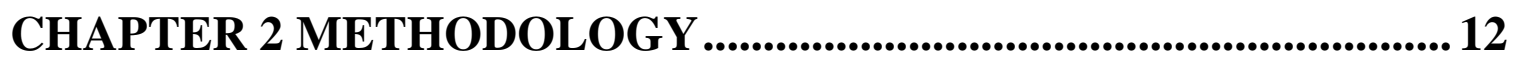

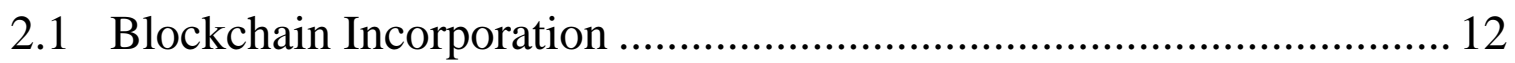

2.1.1 Permission to connect ........................................................... 12

2.1.2 Permission to send, receive or write data.................................... 12

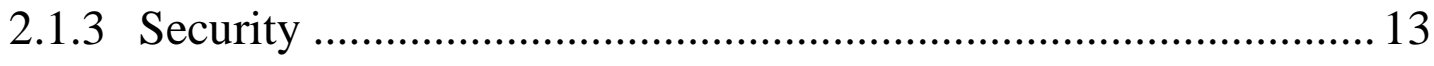

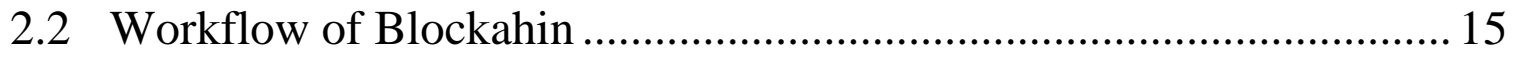

2.2.1. Designing network and allocating IP addresses to hosts ............ 16

2.2.2. Creating the blockchain from the first server .............................. 16

2.2.3. Permissions to connect to the blockchain from next server ........ 16

2.2.4. Creating streams for servers...................................................... 17

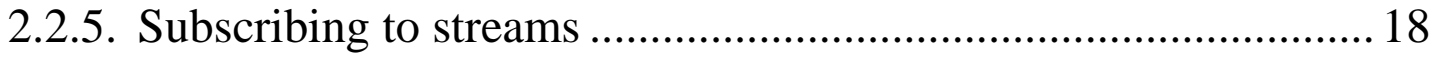

2.2.6. Servers grants permission receive and send data to the stream ... 18

2.3 Data Comparison and Analysis.......................................................... 19 


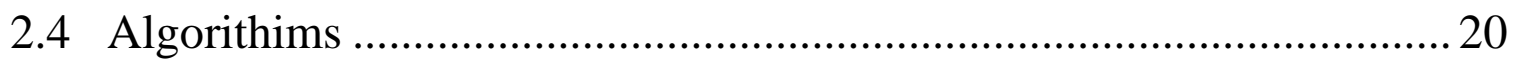

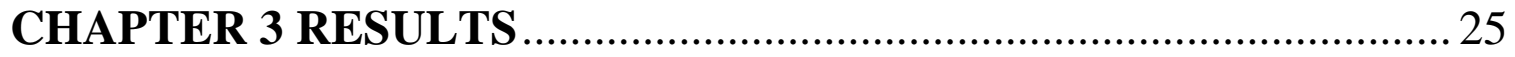

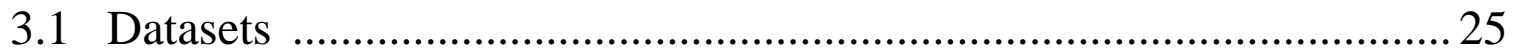

3.2 Feeding Data for making Ideal Data.................................................. 25

3.3 Data Transfer under same Network ….............................................. 26

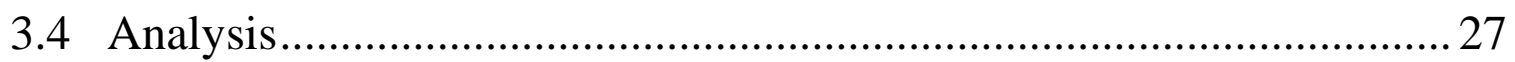

CHAPTER 4 CONCLUSIONS AND FUTURE WORK ........................ 28

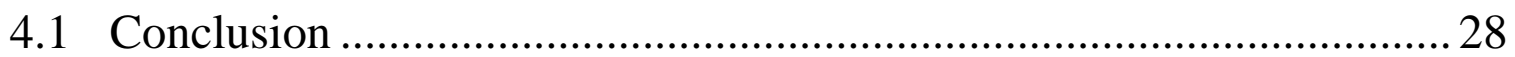

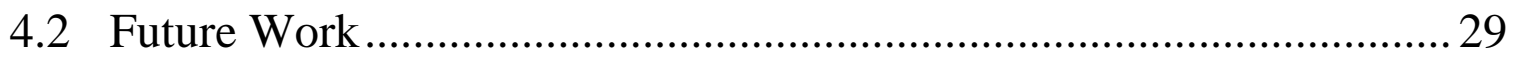

REFERENCES........................................................ 30 


\section{LIST OF FIGURES}

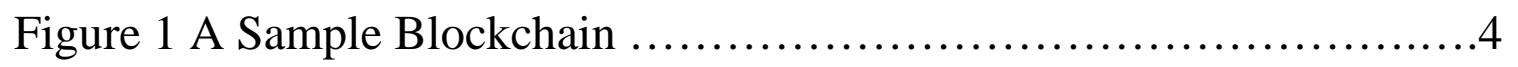

Figure 2 Blockchain Permission workflow.............................15

Figure 3 Data Analysis Work Flow.....................................19

Figure 4 Data VS Time............................................26 


\section{LIST OF ALGORITHMS}

Algorithm 1: Flight_Summary.........................................21

Algorithm 2: File_Upload...........................................22

Algorithm 3:Flie_Receive............................................22

Algorithm 4 Compare....................................................23

Algorithm 5: Result_Upload............................................24

Algorithm 6: Result_Recieve..........................................24 


\section{Chapter 1:}

\section{Literature Review}

The Internet of Things (IOT) refers to the "networked interconnection of everyday objects, which are often equipped with ubiquitous intelligence” [7]. Its purpose is to provide an infrastructure that facilitates "the exchange of "things" in a secure and reliable manner, by overcoming the gap between objects in the physical world and therein information systems" [6]. Objects in the physical world that are inter-networked in the IOT includes vehicles, home appliances, and buildings. These devices are embedded with electronics, software, sensors, and actuators and have network connectivity that allows them to connect to the network.

Due to the technological boom of today, there is a rise in technology-assisted crimes. It may be possible for a hacker to override the electricity in the home and cause equipment to malfunction and create major problems. Therefore, it is absolutely necessary to ensure a physical object to be secured so that it does not malfunction. The IOT is, thus, a seamless network, which uses technology that deals with account security and privacy issues.Bringing devices in IOT is not troublesome but then ensuring the security of these devices in the IOT is what is most important. IOT architecture is a secure ecosystem encompassing all building blocks of IOT architecture. Therefore, it is imperative to understand the different building blocks of IOT, identify the areas of vulnerability that may exist in each 
block. Therefore, exploring the technologies that may be required to amend these weaknesses.

There are a number of ways that includes encrypting the communication between the IOT devices in the network that can be used for securing the IOT network. Encryption can help prevent denial-of-sleep attacks that drain the battery of the device and, also, the distributed denial-of-service (DDoS) attacks.

Recently, a massive distributed denial of service (DDoS) attack struck and crippled the servers of well-known websites like Twitter, Netflix, New York Times, Spotify, GitHub and more. It was reported that "internet users around the world, but mostly in the US, reported that some top websites were not loading” [2]. The attacks were primarily on Dyn - a global DNS service provider. They said that the attacks were "well planned and executed" which came from "tens of millions of IP addresses at the same time" [2] [5]. These were actually IOT devices like printers, DVs, etc which were infected by the Mirai malware.

The case is presented here is to portray that a decentralized approach to IOT networking can solve many of the security issues mentioned above. If a standardized peer-to-peer communication model is adopted which can then be used to process hundreds of billions of transactions between devices. This can significantly reduce costs that are associated with installing and maintaining large centralized data centers. Additionally, it can distribute computation and storage 
needs amongst and across the many devices that make up the IOT networks. Such an approach can help prevent failure in any single node in a network from bringing the entire network to a halting collapse. Gartner - a technology research firm, predicted that by the end of 2017 , more than $20 \%$ businesses will deploy security measures in order to protect their IOT devices and services. IOT devices and service may seem to expand the surface area for cyber-attacks on businesses because the network turns physical objects that used to be offline into online assets which can communicate with enterprise networks. Thus, all businesses whether big or small will have to respond by broadening the scope of their security strategy to include these new online devices.

A decentralized approach to IOT networking would solve many of the issues above. Adopting a standardized peer-to-peer communication model to process the hundreds of billions of transactions between devices will significantly reduce the costs associated with installing and maintaining large centralized data centers and will distribute computation and storage needs across the billions of devices that form IOT networks. This will prevent failure in any single node in a network from bringing the entire network to a halting collapse. Gartner predicted that more than $20 \%$ of businesses will deploy security solutions for protecting their IOT devices and services by 2017, IOT devices and services will expand the surface area for cyber-attacks on businesses, by turning physical objects that used to be offline into 
online assets communicating with enterprise networks. Therefore, a business must respond to this by broadening the scope of their security strategy to include these new online devices.

\subsection{Blockchainand IOT:}

Blockchain was first hypothesized in 2008 and first implemented in 2009. A man named Satoshi Nakamoto was responsible for its invention. From his findings, the blockchain works a "distributed ledger". It can be said that a blockchain is a "public ledger" where all "committed transactions are stored in a chain of blocks" [8]. In order to better understand it, let us take a look at figure 1 below.

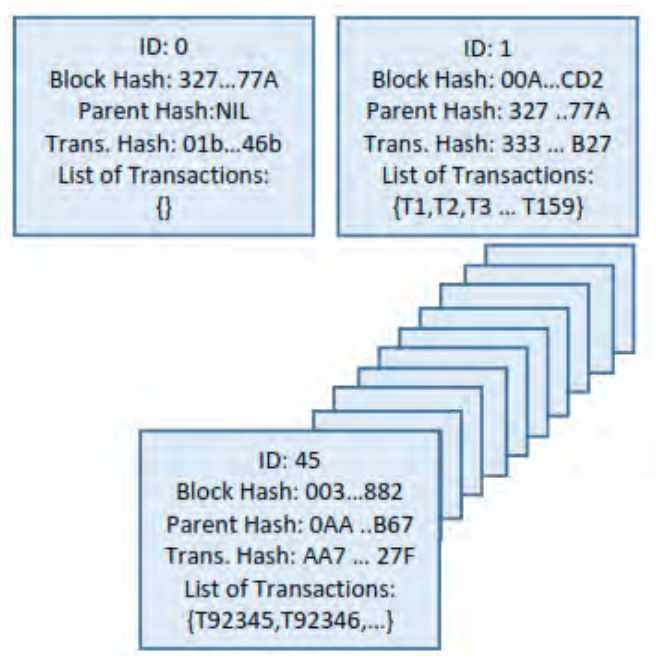

Figure 1: A Sample Blockchain 
The above figure has been taken from the paper, "Blockchain as a Service for IOT: Cloud versus Fog” by Samaniego et al. [5]. This figure is a sample blockchain model for transactions, in it, the valid or verified transactions are stored in the form of a block. This block lists the transactions and each of the blocks is linked to the previous. The blockchain starts with an "initial or genesis block" and as a new block is created, the "hash value" of the previous one is entered. Therefore, bringing any sort of change to the previous block will allow all participants in the subsequent blocks to see it. That is why it can be said thatblockchains are “tamperproof distributed transaction ledgers” [5].

Similar to the figure above, in a blockchain model, the chain grows incessantly when new blocks are added to it. This technology has several key characteristics that include: decentralization, persistency, anonymity, and auditability. Apart from what has been mentioned above, it should also be added that the most interesting feature of the blockchain is that it can work in a decentralized environment. This environment is enabled by "integrating several core technologies such as cryptographic hash, digital signature (based on asymmetric cryptography) and distributed consensus mechanism” [8].

At present, the “distributed ledger” technology has garnered immense interest in the technology sector. The transaction through blockchain occurs in the distributed database of records. This distributed database of records is also known as the 
"public ledger" of all transactions or digital events that have been completed and shared across participating parties. Satoshi Nakamoto not only formulated a hypothesis but also invented the bitcoin along with it. The bitcoin is often called "the first cryptocurrency", is now one of the most popular cryptocurrency in the market as its capital market has reached more than 10 billion dollars in 2016 [8]. The bitcoin is the peer-to-peer version of electronic cash that allows online payments to be sent directly from one party to the other without the need for a third party financial institution such as a bank. In the blockchain module, the digital signatures are validated instead of physical signatures. Additionally, the absence of a third party makes the entire system to be "decentralized”, so, there is no single entity, which might impose any sort of control over the transactions made.

However, there might arise the question that to what extent is this “decentralized” blockchain technology reliable. For bitcoin, Nakamoto himself writes that in order to increase reliability and eradicate the problem that owners did not double-spend the coin in the network, a solution can be presented. The solution, according to him, is to introduce a "trusted central authority", also called a "mint" that checks every transaction for double-spending[3]. Thus, after each transaction has been completed, the coin has to be returned to the mint so that a new coin can be issued. It is only the coins from the mint that can be trusted as coins that are not “double-spent”. So, in order to make it more reliable, the solution proposed by 
Nakamoto to use a protocol implemented by the timestamp server. In the blockchain model, the work of the timestamp server is to take a hash of a block of items, timestamp it and then widely publish the hash. The objective of the timestamp is to prove that the data exists at the time, and as a result, can get into the hash. Each of the subsequent timestamps has the previous timestamp in its hash, thus, forming a "chain, with each additional timestamp reinforcing the ones before it” [3].

Therefore, we can understand that the special nodes in the system called "data miners" provide a "proof of work" which can be fixed to a message, for example, a postage stamp to a postcard. The blockchain relies heavily on hashes and hash functions. Actually, the hash "output" is the result of a conversion of the original information or the "input". Thus, it can be said that the hash function is "a mathematical algorithm that takes an input and transforms it into an output" [4]. This mathematical algorithm of the random number with a prefix of "zeroes” when concatenated, thus, "hashed" contain all the information about the blocks in the blockchain. The function of the cryptographic has is characterized by its extreme difficulty to revert, which, in other words, is to recreate the "input data" from its “hash value alone”. This is known as “collision resistance” [4].

On the other hand, traditional security methods for securing the IOT can be expensive in terms of both energy consumption and processing overhead. 
Additionally, today's state-of-the-art security frameworks are not suitable for IOT because they are "highly centralized" and this difficulty of scale, i.e. "many-to-one nature of the traffic" leads to a "single point of failure" [1]. Thus, use of such security framework may lead to "reveal or noisy data" that will, in turn, hinder the IOT applications personalized services. Therefore, to provide the missing link between user privacy and feasibility in IOT devices, the blockchain model can play an integral role. Tracking connected devices, enabling the processing of transactions and coordinating between them will allow for both reduced costs and security for the IOT industry manufacturers. Therefore, in such model, the blockchain will treat the messages exchanged between the devices similar to the financial transactions occurring in a bitcoin network.

\subsection{Decentralized Data:}

As mentioned previously, the decentralized approach of blockchain technology makes it an interesting choice for connecting IOT devices in the network as the technology has been proven successful in the bitcoin currency mechanism. The basic idea of using a decentralized approach would be to eliminate single points of failure, which will lead to the creation of a more resilient ecosystem for peer-to-peer devices to run on. It is known that every Internet user has access to "public decentralized ledgers". This public nature ensues because in the process of determining "what blocks are added to the chain and what its current 
status is”, everyone participates freely and unconditionally in it [4]. Additionally, as mentioned previously, the decentralized blockchain rests on a "consensus mechanism" of "proof-of-work" which is used for validation purposes. This, in the case of Bitcoin, the "longest chain - the chain with the most proof-of-work - is considered to be the valid ledger” [4].

Therefore, in a fully private ledger, a central locus of decision-making monitors the write-permissions. On the other hand, the read-permissions are either public or restricted [4]. Public and private blockchains are differentiated to the extent to which they are "decentralized" to ensure anonymity. Therefore, the “partially decentralized” or "consortium blockchains” constitute as a hybrid between the "low-trust, i.e. public blockchains" and the "single highly-trusted entity model, i.e. private blockchains” [4]. Blockchains are, indeed, cutting-edge informational devices that can answer different needs and fulfill different objectives. Thus, at present, the resulting way of blockchaining is reliant on a number of organizational and strategic parameters. In these, there exists the "public versus the private" blockchain debate which actually stems down to "a bifurcation between permission and permissionless validators” [4]. Indeed, the blockchain world is an abstract space between the public ledger and private ledger databases but this space is important because it is this decentralized space that acts as “continuum” by which the decentralized data is secured in the whole model. 


\subsection{Secure System:}

Blockchain technology does have the immense potential using which future Internet systems can be constructed. At present, the technical challenges might be there that is creating a hindrance for this to occur. By taking the example of the bitcoin technology, problems that have been associated with the blockchain technology includes the scalability an unethical nature of certain data miners [8]. Thus, to avert problems like these, there needs to be persistence in the blockchain network along with anonymity. That is to say, that, each of the exchanges that are spreading across the network has to be confirmed and recorded "in blocks distributed in the whole network", making it "impossible to tamper" [8]. Additionally, devices in a blockchain network will have a "generated address" using which they can interact with other devices. Multiple addresses can be generated to avoid exposure, and, aforementioned, there is no central party where “private information” is being stored. Thus, this system allows privacy to exist in the data exchanges or the "transactions" that occur.

A number of cryptographic algorithms can be found which is being used in the blockchain technology. Zheng et al. [8]summarize the types of Consensus Algorithms as follows: first is the PoW (Proof of work), which is a consensus strategy used in Bitcoin network [3] and it "requires a complicated computational process in the authentication". Second is the PoS (Proof of stake), which is "an 
energy-saving alternative to POW" because "instead of demanding users to find a nonce in an unlimited space, POS requires people to prove the ownership of the amount of currency”. The third is the PBFT (Practical Byzantine fault tolerance) which is a replication algorithm to tolerate Byzantine faults [4]. Fourth is the DPOS (Delegated proof of stake) which is similar to the POS, where miners get the priority to generate the blocks according to the stakes that they have. The major difference, however, is that "POS is direct democratic while DPOS is representative democratic". Fifth is the Ripple [8] is a consensus algorithm that “utilizes collectively-trusted subnetworks within the larger network". Finally, the Tendermint[8] is a Byzantine consensus algorithm where the new block is determined in a round.

A good consensus algorithm will always be efficient, safe and convenient. At present, consensus algorithms are being devices to solve specific problems in the blockchain model. Upon reviewing multiple kinds of literature, it was found that new consensus algorithms like the PeerCensus, Kraft, Greedy HeaviestObserved Sub-Tree (GHOST) are have been created to in order overcome the limitations. Advantages of the different types of good consensus algorithms include node identity management, energy saving and tolerance power of [8]. All of these different specifications and algorithms will only make the blockchain tamper-proof and as it is decentralized, therefore, no malicious attacks will be able to target it. 


\section{Chapter 2:}

\section{Methodology}

\subsection{Blockchain Incorporation:}

As previously mentioned, blockchain or shared database technology uses the concept of decentralized control over centralized (cloud-based) control as a solution to IOT. When we focus on the disadvantages of using the traditional cloud-based approach for IOT, we have seen that it is, in many ways, insecure and unreliable. Therefore, the features that are included in our model eliminates the drawbacks of the cloud-based system in the following ways:

2.1.1. Permission to connect: Since bitcoin uses a public ledger, hence anybody with an internet connection has access to the shared database of bitcoin. However,blockchain in private networks does not need to have a public database. The blockchain database in private networks should only be viewed by permitted servers/ users because there is no chance of a Sybil attack [11]. Therefore, the issuer of the blockchain chooses to decide which users to include/ not include in the blockchain network.

2.1.2. Permission to send, receive or write data: As blockchain is a decentralized approach, the issuer of the blockchain has the freedom to grant 
administrative privileges to other users/servers such as the right to send, receive or even write data to a blockchain stream.

2.1.3. Security: Since the proposed model works on private networks, where all the entities know each other, hence the concern over security is eliminated.

In our proposed model, we have used few aircraft maintenance parameters (such as airbrake, indicatedangle of attack, lateral engage modes, roll angle lsp, rudder pedal position etc.) and their data (values). The minimum and maximum data outputs of the parameters taken at every minute for the total flight time of an aircraft are considered and their average values are calculated. The mean is compared with the ideal data values of the same parameters to check for any imbalance and these mean values including the result of thecomparisonare uploaded into the blockchain streams, which will be visible to all the servers. Here, in our prototype, we have worked with 4 different servers. Using a virtual machine, we have created 4 different hosts. The servers are made to operate over the same blockchain network and using a bridge network, they are provided with static IP addresses. We have created different streams to store and retrieve data for the individual parameters for each flight. Server-1 holds the ideal data for the parameters in its stream, whereas, both Server- 1 and Server-2 are able to compare the received data with the ideal data. Server-1 acts as a station (Airport-1) server 
for the arrival flight of Aircraft-1(flight-1) and Server-2 acts as another station (Airport -2) for the arrival of another aircraft which is Aircraft-2 (flight-2) where both of these aircrafts are of the same model. The two additional servers maintain and store the in-flight data of flight-1(Aircraft-1) and flight-2 (Aircraft-2) respectively. In our model, we have collected and used the data readings of the component parameters of NASA's X2 test jet plane. We have used the data of every minute for a total of 20 minutes per flight as the sample data to work on. This process can easily be carried out by taking the mean of the in-flight data of an aircraft at a specific time interval (let's say, a minute) for the entire flight duration (excluding take-off and landing) and then compared with the ideal values in order to post the mean data including the comparison results into the blockchain. The data can then be viewed by all the permitted servers in the blockchain network. In this way, if there is any malfunctioning in any of the aircraft components, it can be notified beforehand via the blockchain to the permitted users. This can, thus, reduce the risk of in-flight malfunctioning of the aircraft as the authority concerned will be able to take precautions beforehand. 


\subsection{Workflow of Blockchain:}

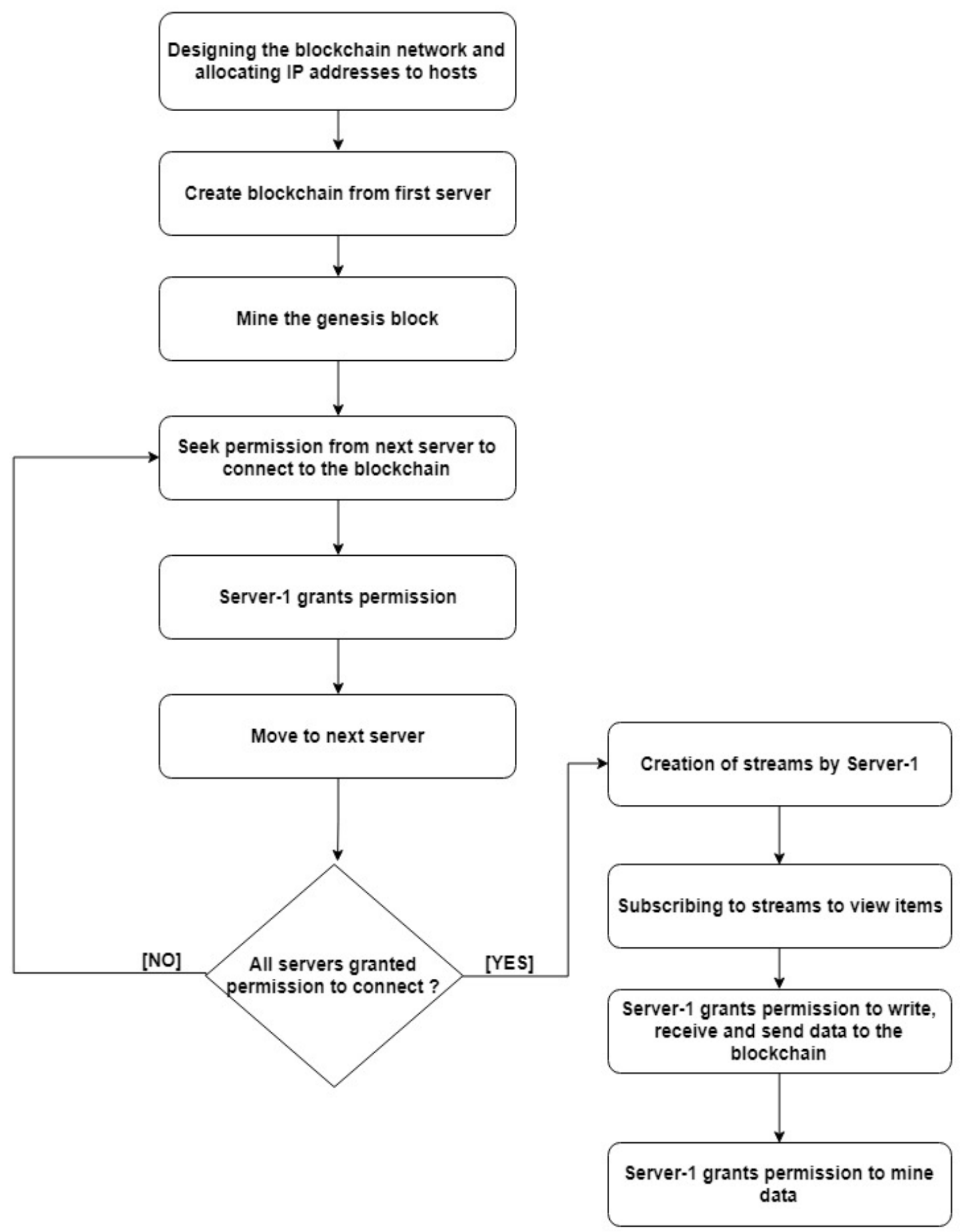

Figure 2:Blockchain Permission workflow 


\subsubsection{Designing the blockchain network and allocating IP addresses to}

hosts: To create the entire blockchain setup, the first task is to choose a network. After the network, in which the hosts will operate, is chosen, static IP addresses from that network are provided to the individual computers. Here, we have ignored the dynamic allocation of addresses to maintain the uniqueness of the servers.

2.2.2. Creating the blockchain from the first server: The first server initializes the blockchain by creating the genesis block. The genesis block is referred to as the first block of a blockchain with no parent. Therefore, it only contains its own hash and does not contain ahash of any previous block. The first server, being the issuer of the blockchain, receives all the administrative privileges by default. The first server has access to view, connect, send, receive and mine data and also has additional privileges of allowing any other node to have administrative access.

\subsubsection{Seeking permission to connect to the blockchain from next server:}

After the genesis block is initialized, other servers will want to access to the blockchain. Since the miner of the genesis block is the sole holder of all administrative privileges, therefore, other nodes need permission from the issuer of the blockchain to connect to and view the chain. Therefore, the next 
server generates a wallet address while connecting to the blockchain, which thereafter, is validated by the issuer and the server can safely connect to view the contents of the blockchain database after the issuer grants the permission to connect. The same process is followed by other servers.

2.2.4. Creating streams for servers: Since we need to store and retrieve data, we created individual streams for each server. The stream of the first server (the creator of the genesis block) contains the ideal data of the aircraft maintenance parameters, (i.e. airbrake, indicated theangle of attack, lateral engage modes, roll angle lsp, rudder pedal position etc.) and is named as ideal. Server 1, afterward, creates two new streams named flight-1 and flight-2 to store the in-flight data of Aircraft-1 and Aircraft-2 respectively. Server- 1 also creates a result stream to store the results of the comparative analysis of the received in-flight data with the ideal data. The data, that can be stored in the streams, are in fact items that are digitally signed by the publishers using their respective private keys. The stream flight- 1 holds the summary (mean) of the in-flight data of Aircraft-1 and the stream flight-2 holds the summary of the in-flight data of Aircraft-2. The resulting stream consists of two unique keys named flight-1 and flight-2. The key flight-1 addresses the comparative data analysis output Aircraft-1, whereas, the key 
flight-2 addresses the comparative data analysis output of Aircraft-2 where both of these aircrafts are of the same model.

2.2.5. Subscribing to streams: Since the first stream, the named ideal contains the ideal data for aircraft maintenance parameters, hence, each server from Server- 1 is given the privilege to view this data. To do this, each server subscribes to the ideal stream. Moreover, Server-2 subscribes to the result stream to view the results of the comparative analysis. However, Servers-3 and Server-4 only subscribe to the keys of the result stream namely flight-1 and flight-2 respectively.

\subsubsection{Server 1 grants permission to write, receive and send data to the} stream:Server-1 grants admin permission to Server-2. Server-1 permits Servers 3 and 4 to write data to flight- 1 and flight-2 streams respectively. Server-2 can publish data to the result stream after performing the data analysis. The resulting stream can be viewed by all the servers. 


\subsection{Data Comparison and Analysis:}

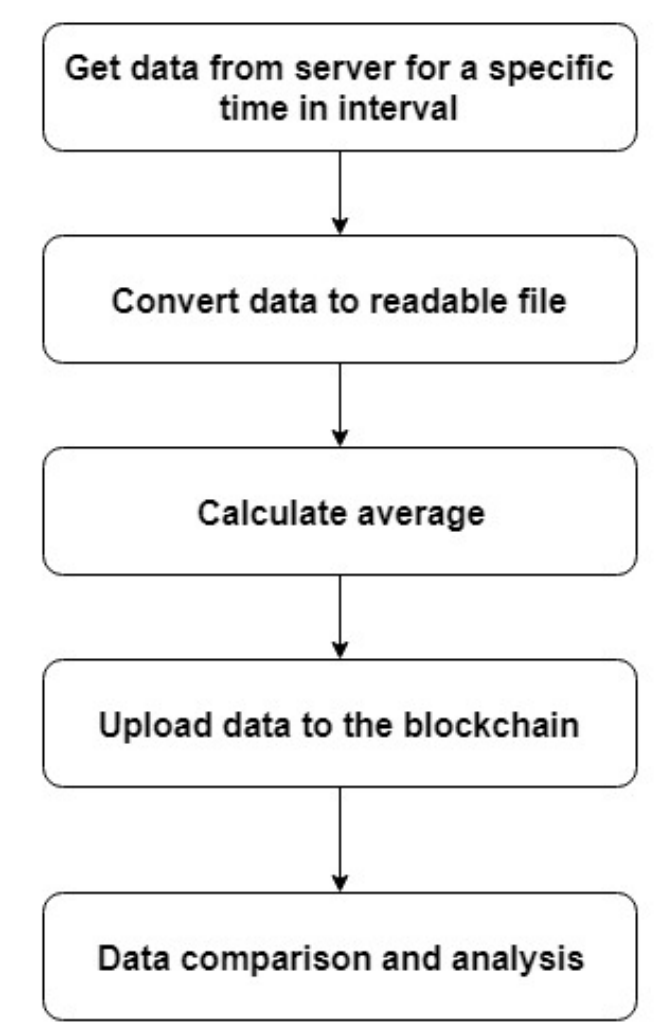

Figure 3: Data Analysis Work Flow

Block 1: Get data from theserver for a specific time interval: As previously mentioned, we have taken the data for aircraft maintenance of NASA's X2 test jet plane. 
Block 2: Convert data to areadable file: The data is converted to areadable format (.txt format) for better understanding. This process makes it easy to analyze the data for further calculations.

Block 3: Calculate average: The data of every minute for a total of 20 minutes (in-flight time) is taken to find out the mean/ average values of both the minimum and maximum threshold of the maintenance parameters.

Block 4: Upload data to the blockchain: The calculated mean of the data is now uploaded to the blockchain streams. In the case of Aircraft-1, the data is uploaded to the flight-1 stream using the private key of the corresponding server. Likewise, the data for Aircraft-2 is uploaded to the flight-2 stream in the same way.

Block 5: Data comparison and analysis: The mean data values of the attributes/ parameters are compared against their ideal values using a comparison algorithm and the results are published to the appropriate key of the result stream by the permitted servers. The output of the comparison is also converted to a readable file that shows which of the aircraft components have readings within the ideal range and which components have readings deviated from the ideal values.

\subsection{Data Algorithms:}

As mentioned earlier, before running all the necessary algorithms we have first read the data from the system and converted them into areadable file format (.txt) for 20 minutes in-flight time (excluding landing and takeoff). 
After completing this step, the following algorithms and pseudo codes are followed.

\section{Algorithm 1:Flight_Summary}

Create lists for max_val, min_val, attribute

Read $\leftarrow$ flight_log[1]

for $\mathrm{x}:=0$ to number of attributes of aircraft

begin

$$
\begin{aligned}
& \max \_v a l:=\text { find }(\max [x], \text { from flight_log[1]) } \\
& \min \_v a l:=\text { find }(\min [x], \text { from flight_log[1]) } \\
& \text { list := append (max_val, min,val, attribute) }
\end{aligned}
$$

end;

for $y:=2$ to total number of data log files of aircraft begin

$$
\begin{aligned}
& \text { Read } \leftarrow \text { flight_log[y] } \\
& \text { forz: }=0 \text { to number of attributes of aircraft } \\
& \text { begin } \\
& \text { max_val := max_val[z] + find(max[z] ,from flight_log[y]) } \\
& \min \_v a l:=\min \_v a l[z]+\text { find}(\min [\mathrm{z}] \text {,from flight_log[y]) } \\
& \text { list := append (max_val, min,val, attribute) } \\
& \text { end }
\end{aligned}
$$

end 
For making the flight summary for specific flights we have used this "Flight_Summary" algorithm where we have basically make an average of the maximum and minimum values of each attribute for the total 20 minutes in-flight time.

\title{
Algorithm 2:File_Upload
}

connect $\rightarrow$ blockchain

subscribe(stream_name)

read $\leftarrow$ flight_log_summary

encrypted_data := encode_Data(Read)

publish(encrypted_data,flight_key)

print ("Flight data has been uploaded successfully into the blockchain")

The aircrafts will use the above algorithm to upload the data to the blockchain so that the airports can view and retrieve the in-flight data to make necessary calculations for producing the final maintenance report for that flight

\author{
Algorithm 3: Flie_Receive \\ connect $\rightarrow$ blockchain \\ subscribe(stream_name) \\ read $\leftarrow$ data_from_stream(flight_stream,flight_key) \\ data $:=\operatorname{decode}(\mathrm{read})$ \\ store(data) $\rightarrow$ flight_log \\ print ("Flight data has been received successfully")
}


The "File_Receive" algorithm is used by the airports to view and retrieve the inflight data for a particular flight. After receiving the data, the airports will make necessary calculations to produce the desired output.

\section{Algorithm 4: Compare}

real_ideal_max ,real_ideal_min := ideal_data_list(ideal_data_summary)

real_flight_max, real_flight_min := flight_data_list(flight_log)

forx, $\mathrm{k}:=0$ to number of attributes of aircraft

if ( real_flight_max[x] >real_ideal_max[x] or real_flight_min[x]

$<$ real_ideal_min[x])

print "(Warning !!! value of this attribute[k] is out of range")

else

print( "attribute[k] is working perfectly")

end

store(data)

To make a comparison between the ideal data and flight data we have used this algorithm. This also prepares the final output which will be needed for the maintenance of the aircraft. 


\section{Algorithm 5:Result_Upload}

connect $\rightarrow$ blockchain

subscribe(stream_name)

read $\leftarrow$ result_summary

encrypted_data := encode_Data(Read)

publish(encrypted_data,flight_key)

print ("Flight result has been uploaded successfully into the blockchain")

The airports will use the "Result_Upload" algorithm to upload the final maintenance result into the blockchain so that the specific aircraft can identify the faulty systems and also upon the arrival of that flight into that airport it will help the maintenance engineers to fix those issues very swiftly and efficiently.

The aircrafts will use this algorithm to retrieve the flight maintenance result prepared by the airport.

\section{Algorithm 6: Result_Receive}

connect $\rightarrow$ blockchain

subscribe(stream_name)

read $\leftarrow$ data_from_stream(result_stream,flight_key)

data $:=\operatorname{decode}(\mathrm{read})$

store(data) $\rightarrow$ result_log

print ("Flight result has been received successfully") 


\section{Chapter 3:}

\section{Results}

\subsection{Datasets:}

All the datasets for this thesis were collected from NASA's Dashlink project where they have provided Data for X'2 jet [8]. All these data were collected as MAT lab formatted and changed to python's duct structure for experimental convincing of the use of the data. In this dataset, 10GB of data were received where for simulation roughly 2 GB of data was needed. From each file, we were able to get all readings taken by machines at that particular time. Where each set of data were roughly on an average of $10 \mathrm{MB}$ and maximum about $20 \mathrm{MB}$ and minimum ranging from about 2MB.

\subsection{Feeding Data for making Ideal Data:}

From the following 2GB of data, for thecreation of ideal data set to compare the flight data with, one of the machines were given roughly 1.5 GB of data so that the machines can generate a data set with which a reliable data to compare with is generated. For this, over 150 minutes of on flight data were compared and used in making the ideal data. Where it able to understand which data under normal circumstance were off and the ones which need to be checked. 


\subsection{Data Transfer under thesame Network:}

from the simulation, after every 1 minutes the data is sent to the airports from the aircrafts. At each interval, a maximum of $64 \mathrm{MB}$ can be sent. Therefore, if any more than that it needs to be sent a packet of 64MB. However, the data set neverexceeded

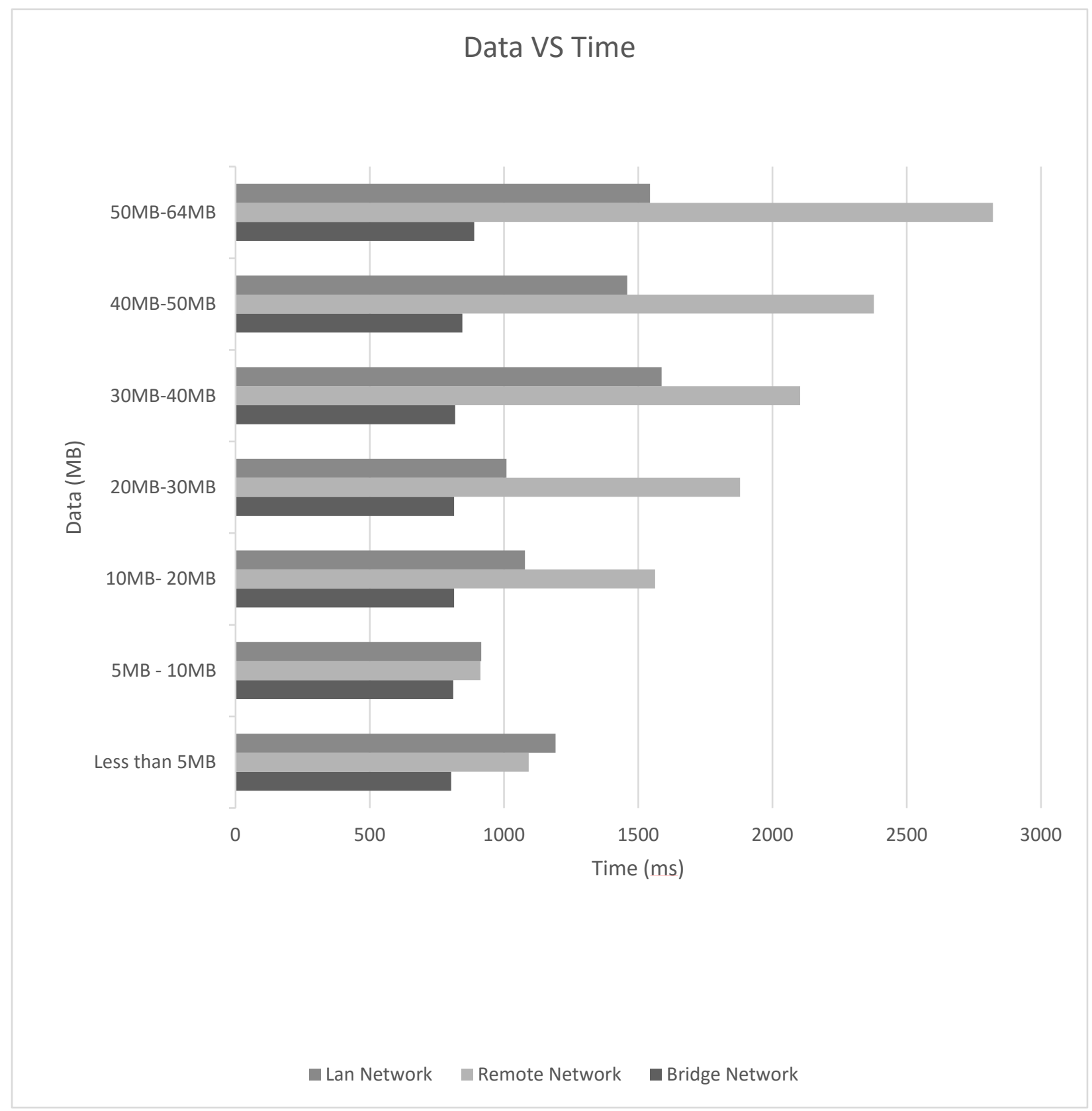

Figure 4: Data VS Time 
more than $64 \mathrm{MB}$ during the simulation,therefore, all the data for that particular minute is sent at once. Moreover, time needs to upload the file take less than $1000 \mathrm{~ms}$ in thebridge network.

On the other hand, if the data need to be sent remotely under the same network it takes roughly over 2000ms. However, nodes which are outside the network is a different case altogether. As blockchain works on something peer to peer network. It is evident that, if no nearby peer node is available then data becomes hard to come by. This problem can easily solve by using the nodes of theairport as aserver. As theserveris intended to do so they are always running for which every airport will always be used,therefore, no nodes that have a reservoir of data will be always in the network and can send data to other nodes when needed from the closest node nearby.Moreover, it is also important to note that the mining time needed by the miners are 500ms.

\subsection{Analysis:}

After the data received by the servers i.e. the Airports they accumulate 20 minutes' worth of data at once and then compare itself with the ideal data which have been feed to the machine. After the range is compared with the data, we can identify where or what part of the plane needs to be checked. The Accuracy rate of the comparison is $82 \%$ as we analyzed the same data for 50 times from that we get about 41 times we get almost same or very close data from the previous one. 


\section{Chapter 4:}

\section{Conclusion and Future Work}

\subsection{Conclusion}

In our proposed model objective is to make network model in which IOT device can transfer data through adecentralized network instead of a centralized network. Moreover, for the transferring of data and to replicate a real-life scenario we choose aircraft and airport communication of engine and machine data transfer as such data are easily intercepted by foreign and unauthorized party [9]. In such a scenario we see that crypto hash function of used by blockchain, giving every node unique wallet address and forming TXID after every transaction that takes place in the blockchain which is transmitted to all server like nodes in the network, makes it very difficult to hack or penetrate such a system even after such hacking is done, the concept of decentralized network prevents further damage if any data is tried to modify. Moreover, for one aircraft there is at least two stakeholders these are the reason blockchain is more secure than theconventional network. Moreover, when have a centralized network, it is easy to DDOS attack that server if every airport in the network is a server it becomes virtually impossible to modify any such data. For such reason, we believe that with further research under blockchain we can make a network which over time will become impenetrable. 


\subsection{Future Work}

In this paper, we have worked with a virtual network using virtual machines, however, this needs to be tested over a vast network to make sure the security of the network is refined. Moreover, the data we tested this model was of a jet planes, hence, we need to use a commercial jet's datato test in such a system as well. The analysis we made can be done more accurately by introducing the concept of machine learning and data mining, which can predict where there may be issues with the aircraft and make maintenance time quite short. 


\section{References}

[1]Dorri, A., Kanhere, S. S., Jurdak, R., \&Gauravaram, P. (2017, March). Blockchain for IoT security and privacy: The case study of a smart home. In Pervasive Computing and Communications Workshops (PerCom Workshops), 2017 IEEE International Conference on (pp. 618-623). IEEE.

[2] Leswing, K. (2016). A massive cyberattack knocked out major websites across the internet. Business Insider, 21.

[3] Nakamoto, S. (2008). Bitcoin: A peer-to-peer electronic cash system.

[4] Pilkington, M. (2015). Blockchain technology: principles and applications.

[5] Samaniego, M., \& Deters, R. (2016, December). Hosting virtual IoT resources on edge-hosts with blockchain. In Computer and Information Technology (CIT), 2016 IEEE International Conference on (pp. 116-119). IEEE

[6] Weber, R. H., \& Weber, R. (2010). Internet of Things: Legal Perspectives, vol. 49. Xia, F., Yang, L. T., Wang, L., \&Vinel, A. (2012). Internet of things. International Journal of Communication Systems, 25(9), 1101.

[7]Zheng, Z., Xie, S., Dai, H. N., \& Wang, H. (2016). Blockchain challenges and opportunities: A survey. Work Pap.

[8]Flight Data For Tail 663 A dataset shared by Bryan Matthews https://c3.nasa.gov/dashlink/projects/85/ 
[9] Cyber Threats against the Aviation Industry ICS SECURITY ON APRIL 8, 2014

http://resources.infosecinstitute.com/cyber-threats-aviation-industry/\#gref

[10] Christidis, K., \&Devetsikiotis, M. (2016). Blockchains and smart contracts for the internet of things. IEEE Access, 4, 2292-2303.

[11] Greenspan, G. (2015). MultiChain Private Blockchain-White Paper. 